\title{
Fragmentation and cooling of doubly charged anthracene studied in an electrostatic storage ring
}

\author{
S. Martin, ${ }^{1, *}$ L. Chen,,${ }^{1,2}$ A. Al-Mogeeth, ${ }^{1}$ and J. Bernard ${ }^{1}$ \\ ${ }^{1}$ Institut Lumière Matière, UMR 5306 Université Lyon 1, Centre National de la Recherche Scientifique, Université de Lyon, \\ 69622 Villeurbanne Cedex, France \\ ${ }^{2}$ Institute of Modern Physics, Department of Nuclear Science and Technology, Fudan University, Shanghai 200433, China
}

(Received 17 October 2018; published 25 January 2019)

\begin{abstract}
The cooling of anthracene dications $\left(\mathrm{C}_{14} \mathrm{H}_{10}\right)^{2+}$ has been studied in a time range of $10 \mathrm{~ms}$. Intact $\left(\mathrm{C}_{14} \mathrm{H}_{10}\right)^{2+}$ ions were produced in a Nanogan electron cyclotron resonance (ECR) ion source and stored in a compact electrostatic ion storage ring, the Mini-Ring. The internal energy distribution of the stored dications was probed every millisecond during the storage time using optical parametric oscillator (OPO) nanosecond laser pulses. The wavelength of the laser was tuned at $560 \mathrm{~nm}(2.214 \mathrm{eV})$, which is the center of a broad resonance peak attributed to the $S_{2}-S_{0}$ transition of $\left(\mathrm{C}_{14} \mathrm{H}_{10}\right)^{2+}$. The fast decay of neutral fragment counts due to laser induced dissociation was recorded for several probing times. These experimental neutral decay curves were reproduced by a model that accounted for the slow evolution of the internal energy distribution of the stored ions due to dissociation and radiative cooling processes. From the simulated internal energy distributions, we have estimated an energy shift rate varying from 140 to $30 \mathrm{eV} / \mathrm{s}$ in the time range from 1 to $9 \mathrm{~ms}$, which was attributed mainly to the radiative cooling due to delayed electronic transitions. The electronic fluorescence rate was estimated to $331 \mathrm{~s}^{-1}$ for an internal energy of $7 \mathrm{eV}$, which was similar to the electronic fluorescence rate of an anthracene cation at the same energy. We conclude that, for anthracene, the fluorescence cooling process is not sensitive to the charge of the molecule for low charge states, cation and dication.
\end{abstract}

DOI: 10.1103/PhysRevA.99.012712

\section{INTRODUCTION}

In the last decade, many studies have been devoted to the evolution of the internal energy distribution (IED) of highly isolated polycyclic aromatic hydrocarbon (PAH), linear hydrocarbons $\left(\mathrm{C}_{n} \mathrm{H}_{m}\right)$, fullerene, and metallic cluster molecules [1-6]. The knowledge on the IED concerning the PAHs is of particular interest in the estimation of the minimum size of stable PAH molecules irradiated under UV in the interstellar medium (ISM) [7]. Notably, laboratory investigations on fast and slow dissociation and cooling processes at different excitation energies are badly needed to support astrophysical models.

Experiments using recent electrostatic storage rings (ESRs) are suited to measure slow processes such as delayed dissociation, cooling by infrared (IR), and delayed fluorescence emission. The evolution of the IED of molecules can be measured using the "action spectroscopy" technique over a large range from microseconds to seconds especially with cryogenic ESR rings $[3,8]$. It is well known that hot molecules release their internal energy mainly by dissociation and radiative processes. For small PAH cations, according to theoretical prediction [9], dissociation processes are expected to be dominant at a high internal energy range, typically higher than $7 \mathrm{eV}$ in the case of anthracene. In the very low internal energy range, the molecules are cooled down mainly by IR photon emissions. In the intermediate-energy range, where the radiative decay starts to compete with the dissociation process

\footnotetext{
*smartin@univ-lyon1.fr
}

and becomes the dominant energy release mechanism with decreasing internal energy, the delayed fluorescence process (DFP) is expected to be dominant over the IR emission process (in the range from 5 to $7 \mathrm{eV}$ for anthracene). This DFP (also referred to as Poincaré fluorescence, recurrent fluorescence, or fluorescence from thermally excited electronic states, depending on the situations and on the authors), first predicted in an early theoretical work [10], involves inverse internal conversion (IIC) from high vibrational levels of the ground electronic state to low-lying electronic states, leading to a fluorescence emission in the visible range. In recent papers, we have provided experimental evidence showing that the DFP was indeed a very efficient radiative cooling process compared to the cooling via infrared emission for naphthalene [11] and anthracene [12] cation molecules in the intermediateenergy range. This process, not very well known up to now, has to be taken into account, for instance, in the modeling of the size distribution of PAHs in the ISM.

In this paper, we are interested in the charge effect on the cooling process of PAH molecules. Doubly charged PAHs might also be formed by UV photoionization in the ISM. However, laboratory studies on such species are still very rare. Indeed, the production of intact dications at low- and intermediate-energy ranges, the storage of such molecules, and the probe of the evolution of the IED are challenging tasks. In this paper, we report an experimental investigation on the DFP for the anthracene dication. Intact $\left(\mathrm{C}_{14} \mathrm{H}_{10}\right)^{2+}$ ions were produced in a Nanogan electron cyclotron resonance (ECR) ion source and stored in a compact electrostatic ion storage ring, the Mini-Ring. The internal energy distribution of the stored dications was probed during the storage time 
with optical parametric oscillator (OPO) nanosecond laser pulses. Using experimental and analysis methods similar to those reported in our earlier works, we have estimated the DFP electronic fluorescence rate of anthracene dications. It was compared with the values obtained for anthracene cations in order to discuss the charge effect on the radiative cooling of anthracene.

\section{EXPERIMENTAL SETUP}

The experimental setup has been described in detail in previous papers $[12,13]$. Here, we give a very brief description of the setup. The small ESR used in this experiment, called Mini-Ring, is shown in Fig. 1. It consists of four parallel plate deflectors and two electrostatic conical mirrors. The anthracene dications were produced in an ECR ion source which was optimized for the formation of intact $\left({ }^{12} \mathrm{C}_{14} \mathrm{H}_{10}\right)^{2+}$ molecules. The $\left({ }^{12} \mathrm{C}_{14} \mathrm{H}_{10}\right)^{2+}$ ion beam was extracted, accelerated to $12 \mathrm{keV}$, and selected without ambiguity using a $90^{\circ}$ magnet. To exclude the possible contribution in the selected $\left({ }^{12} \mathrm{C}_{14} \mathrm{H}_{10}\right)^{2+}$ peak from singly charged fragments of half the mass of anthracene, experiments have been also performed with dications of the isotope molecule $\left({ }^{12} \mathrm{C}_{13}{ }^{13} \mathrm{C}_{1} \mathrm{H}_{10}\right)^{2+}$ which was not blended with any singly charged fragments. In the two cases we obtained very similar decay spectra showing that possible contributions due to singly charged fragments resulting from symmetrical dissociation of anthracene were completely negligible when the ECR source was optimized for $\left({ }^{12} \mathrm{C}_{14} \mathrm{H}_{10}\right)^{2+}$ ions. The ion beam was chopped into bunches of $6 \mu \mathrm{s}$ in time width every $10 \mathrm{~ms}$ and stored in the Mini-Ring with a revolution period of $4.6 \mu$ s. The ring was nearly filled up with anthracene dications, except for a very small part due to the short time necessary for closing the ring after the ion bunch injection. After a few milliseconds, due to the energy and trajectory dispersions of the beam, the ion density was nearly constant along the ring with only a small shallow density hole. The storage lifetime of an ion bunch was limited by collisions with residual gas molecules under high vacuum conditions in the ring chamber $\left(2 \times 10^{-9} \mathrm{mbar}\right)$. It was measured to be about $200 \mathrm{~ms}$, a slightly shorter time compared to the storage lifetime of singly charged anthracene cations measured to $300 \mathrm{~ms}$ in our previous works $[12,14]$. This is in qualitative agreement with the fact that for dications a larger electron capture cross section is expected in collisions with neutral residual gas molecules than for cations. The storage cycle of $10 \mathrm{~ms}$ was chosen much shorter than the storage lifetime. Ion losses due to collisions with residual gas were therefore neglected in further analysis.

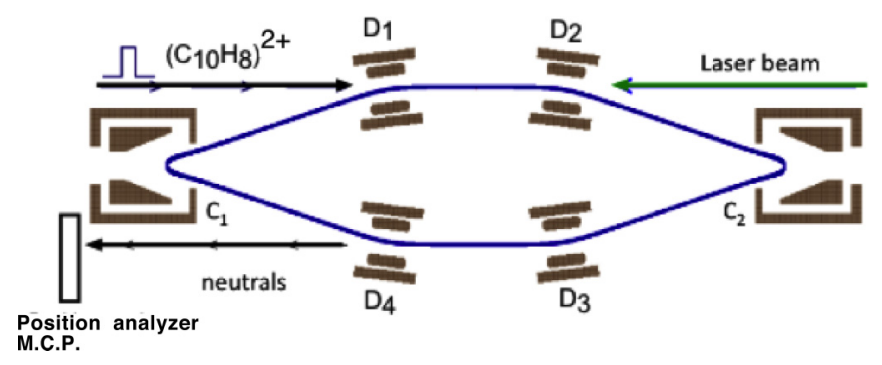

FIG. 1. Experimental setup.
As shown later in this paper, the dominant channels for the dissociation of anthracene dication are the emission of $\mathrm{C}_{2} \mathrm{H}_{2}$ and $\mathrm{H}$ neutral fragments. Small and heavy charged fragments are not detected in this version of the Mini-Ring. Neutral fragments resulting from dissociation events between $\mathrm{D}_{3}$ and $\mathrm{D}_{4}$ (Fig. 1) were detected by a multichannel plate (MCP) detector placed close to the electrostatic mirror $\mathrm{C} 1$. A nanosecond laser (second harmonic or OPO output of Nd:YAG EKSPLA NT242) beam, operated at low power typically less than $30 \mu \mathrm{J} /$ pulse, overlapped the ion beam between $D_{1}$ and $D_{2}$ in merging parallel beam configuration. The sizes of the laser and ion beams have been estimated to be about $2 \mathrm{~mm}$ in diameter. Special care was taken to reduce the betatron oscillation of the ion beam [13]. It could be considered that the geometrical overlap between the laser and ion beams was constant over the whole storage time. The repetition frequency of the laser was $1 \mathrm{kHz}$. Therefore, during a storage cycle of $10 \mathrm{~ms}$, nine laser pulses merged the ion beam, giving an enhanced neutral signal each millisecond. The laser frequency and the delay between the injection of the ion bunch and the first laser pulse were adjusted so that each of the nine laser pulses irradiated roughly a different population of the stored ions. It has been verified that for a given laser excitation time $t_{\text {laser }}$, using either multi or single laser pulse mode, no significant difference was observed on the laser induced neutral measurements in the present range of OPO laser energies (typically $30 \mu \mathrm{J} /$ pulse). The multiple laser pulse mode had the advantages to save experiment time and to record the nine laser induced decays in the same experimental conditions with negligible drawback effects such as a possible reheating of the anthracene dications.

\section{DATA ANALYSIS AND DISCUSSION}

As mentioned before, neutral $\mathrm{C}_{2} \mathrm{H}_{2}$ and $\mathrm{H}$ fragment emissions are the dominant dissociation channels compared to the loss of charged fragments for the dissociation of $\left({ }^{12} \mathrm{C}_{14} \mathrm{H}_{10}\right)^{2+}$ as measured in Ref. [15]. Dissociation rates versus internal energy for cation and dication have been studied previously in Ref. [16]. The microsecond dissociation lifetime corresponds to internal energies of 11 and $10 \mathrm{eV}$ for the cation and dication, respectively, showing a strong charge effect in the stability of the anthracene molecule in this energy range. The dissociation rate $k_{\text {diss }}$ for anthracene dications with internal energy $E$ was determined using a statistic dissociation model following the Arrhenius law. We have used the same preexponential factor $A=2 \times 10^{16} \mathrm{~s}^{-1}$ and dissociation energy $E_{d}=3 \mathrm{eV}$ as those found in Ref. [16]. The values used here are different from those reported in Ref. [15] $\left(A=2 \times 10^{14}\right.$, $E_{d}=3 \mathrm{eV}$ ), where there was a calibration error. Indeed, in the calibration procedure in Ref. [15], we used the singlet instead of the triplet transition of $\mathrm{Ar}^{2+}$ (spin conservation rule in the collision with the fluorine cation) resulting in a shift of $1.7 \mathrm{eV}$ toward higher excitation energy.

As a preliminary experiment, we recorded a photodissociation spectrum of $\left({ }^{12} \mathrm{C}_{14} \mathrm{H}_{10}\right)^{2+}$ (Fig. 2). Neutral fragment counts resulting from laser induced dissociation have been recorded versus the photon energy. In order to improve the signal to noise ratio, the storage time was reduced to $2 \mathrm{~ms}$ and only one laser pulse was sent into the ring with about 


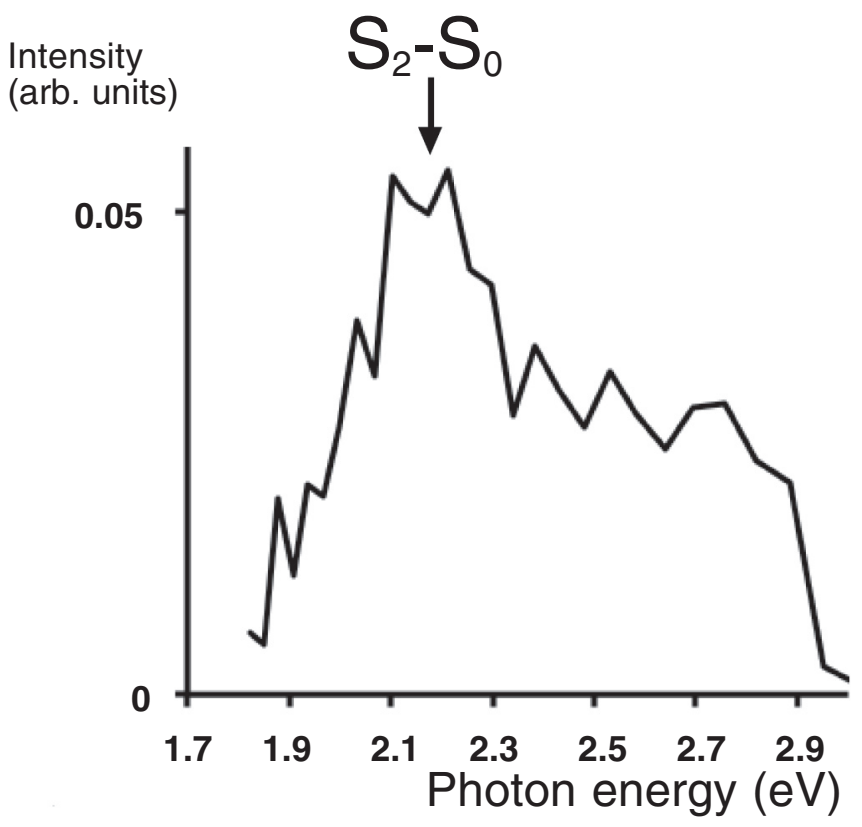

FIG. 2. Photodissociation spectrum of the anthracene dication. Here, the laser pulse time $t_{\text {laser }}$ is $1 \mathrm{~ms}$ and the storage time is reduced to $2 \mathrm{~ms}$.

1-ms delay time. Careful corrections of the laser intensity as a function of the wavelength and of fluctuations of the ion beam have been made. In Fig. 2, a broad line is observed around $2.2 \mathrm{eV}$ at $560 \mathrm{~nm}$. This resonance line is attributed to the $S_{2}-S_{0}$ transition, because it is close to the calculated value of $2.45 \mathrm{eV}$ corresponding to the $S_{2}-S_{0}$ transition of the dication anthracene from the database using the density functional theory of Malloci et al. [17]. According to their calculation, this transition has a rather high oscillator strength of 0.206 . In the following, we focus only on results obtained with a laser excitation tuned at the center of this main peak.

The spectrum in Fig. 3 was obtained with the multipulse laser excitation mode. The neutral counts detected with the

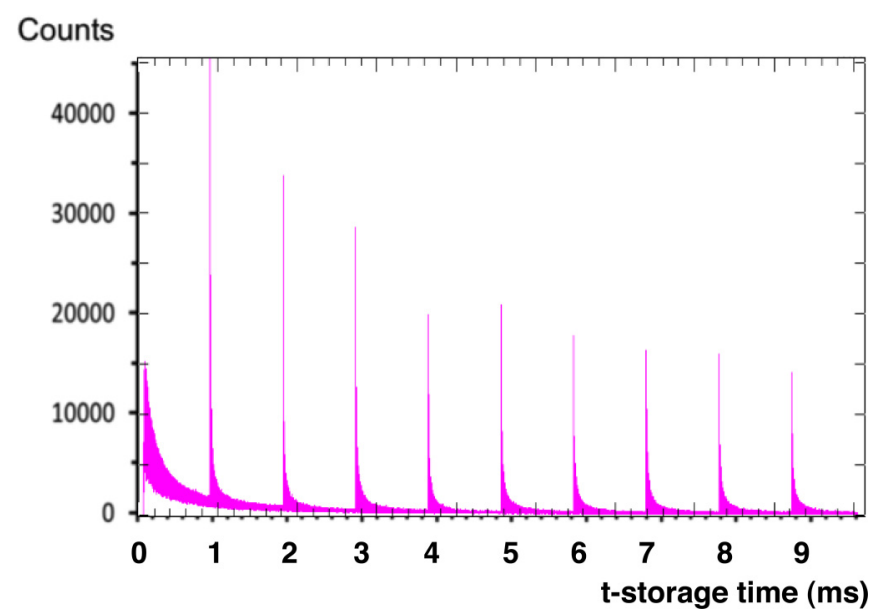

FIG. 3. Neutral yields due to the photodissociation of anthracene dications with OPO laser irradiation $(560 \mathrm{~nm})$ pulses at $t_{\text {laser }}$ from 1 to $9 \mathrm{~ms}$ (1-ms steps).

\section{Counts}

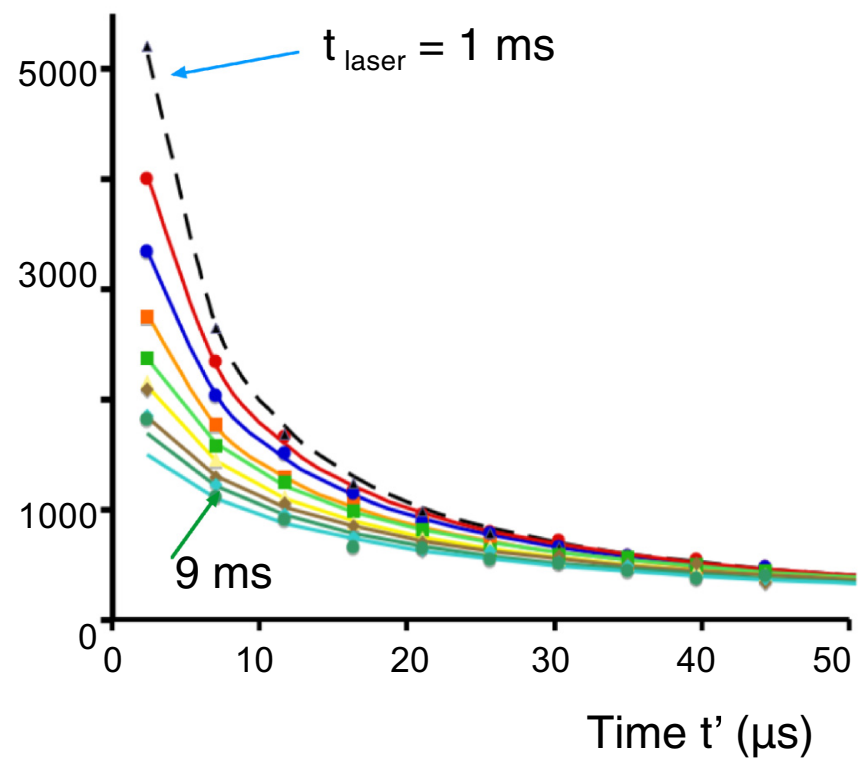

FIG. 4. Experimental (symbols) and simulated (plain lines) neutral decay curves as a function of the storage time $t^{\prime}$ with respect to $t_{\text {laser }}$ for $t_{\text {laser }}=1$ to $9 \mathrm{~ms}$.

MCP were plotted as a function of time from $t=25 \mu$ s to $10 \mathrm{~ms}$. The trigger signal of the beam chopper was taken as the reference of $t$. Before $t=1 \mathrm{~ms}$, the detected neutral fragments were mainly due to the so-called natural decay of hot anthracene dications, i.e., the dissociation of dications that have been produced with high internal energy in the ECR source. The contribution of this natural decay extends up to $3 \mathrm{~ms}$. It is estimated to be about $17 \%$ of the total population of the stored ions. From 1 to $10 \mathrm{~ms}$, nine laser induced decays are observed in Fig. 3. Although the signal to noise ratio is very high, the population of ions excited and dissociated due to laser excitation represents only a very small part of the total stored population. Therefore, the density of stored ions can be considered approximately constant in the range from 3 to $10 \mathrm{~ms}$. The maximum intensity of the decays decreases regularly from 1 to $9 \mathrm{~ms}$, except for the decay at $4 \mathrm{~ms}$ for which the maximum intensity is slightly lower than that of the adjacent decay at $5 \mathrm{~ms}$. This unexpected irregular behavior was due to the fact that the laser pulse at $4 \mathrm{~ms}$ merged a section of the bunch including the shallow density hole. A correction was made in order to account for this problem. The regular decrease of maximum intensity with the laser excitation time $t_{\text {laser }}$ is a first indicator that the IED of the stored ions evolves with the storage time. Indeed, if the IED does not evolve substantially, constant neutral decays should be expected whatever the laser excitation time. This was actually observed in many cases as the naphthalene dimer cations due to the absence of an efficient cooling mechanism (to be published in a forthcoming paper).

Using the same procedure as in Martin et al. [12], the spectrum shown in Fig. 3 was converted to nine decay curves displayed in Fig. 4. After laser excitation through $\mathrm{D}_{1}$ and $\mathrm{D}_{2}$ at $t_{\text {laser }}$ (from 1 to $9 \mathrm{~ms}$ ), the total neutral count recorded each time the ions fly through $\mathrm{D}_{3}$ and $\mathrm{D}_{4}$ in the ring was 


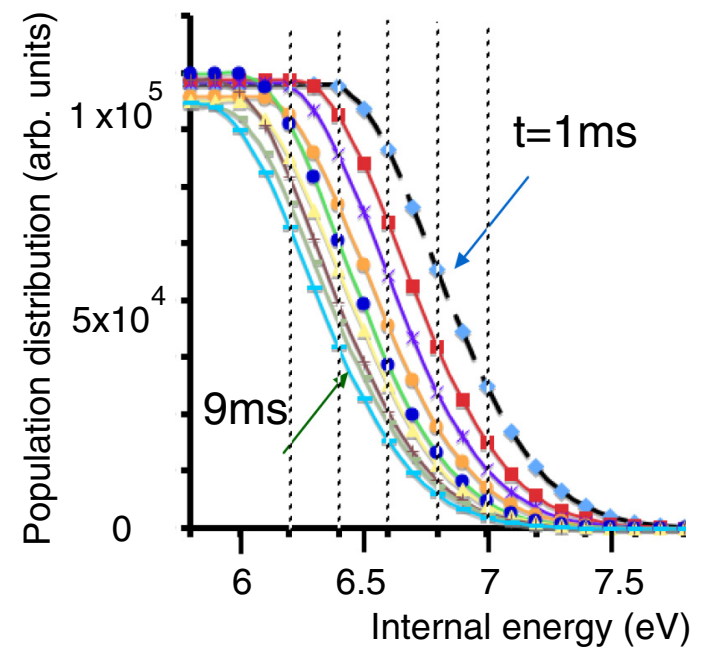

FIG. 5. Simulation of the IEDs after subtraction of the laser photon energy (here $2.214 \mathrm{eV}$ ) vs the storage time from 1 to $9 \mathrm{~ms}$ (1-ms steps).

plotted as a function of $t^{\prime}=t-t_{\text {laser }}, t$ corresponding to the center of each very narrow peak in Fig. 3. Corrections of the background counts and of beam density fluctuations (see Ref. [12] for details) were made. The count around $t^{\prime}=2.3 \mu \mathrm{s}$ corresponds to the measurement at the first half turn after laser excitation. With increasing $t_{\text {laser }}$, this first point decreases regularly and the curves decay more slowly. These features are similar as in our previous observations in other small PAH molecules, naphthalene and anthracene cations [11,12]. In these cases, slower neutral decays were also correlated with longer storage times, i.e., larger $t_{\text {laser }}$, and it was interpreted as a more sensitive indicator of the evolution of the IED.

Following Ref. [12], for each $t_{\text {laser }}$ time, the corresponding IED after laser excitation was simulated using a statistical dissociation model in order to reproduce the measured decay curves. Here, we do not test the validity of the model but we use it to extract parameters of the shape of IED. To start a simulation, a half-Gaussian function with $\sigma=0.6 \mathrm{eV}$ was used as the initial energy distribution shape. As we have seen in the analysis of decays for anthracene cations, this shape was able to reproduce correctly the experimental decay curves, especially the value at $2.3 \mu \mathrm{s}$. Each of the decay curves of Fig. 4 was fitted independently. The free parameters in the modeling were the maximum and the center of the half-Gaussian function of each IED (see Ref. [12] for details). We have found that the best value for $\sigma$ was $0.6 \mathrm{eV}$ for all decay curves and this value was fixed and used in the final adjustment to fit the nine decays curves. As in the analysis of anthracene cations, we have tested various shapes of IED including a simple Gaussian shape. The results show that the fit is not very sensitive in the low-energy range (less than

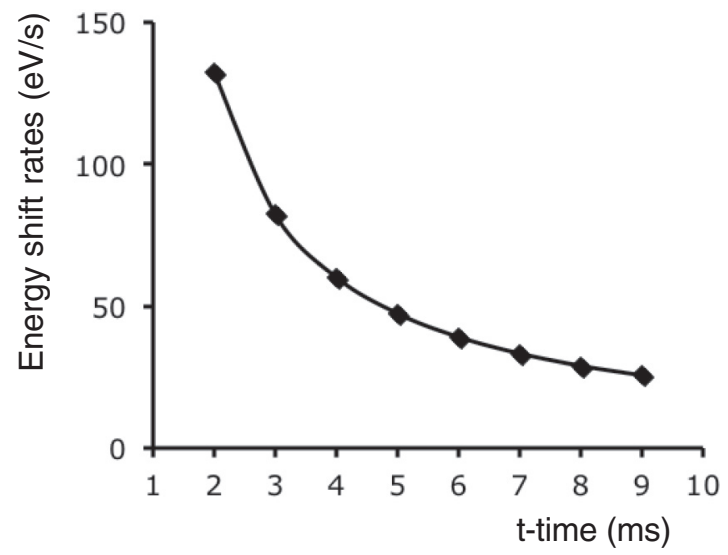

FIG. 6. Measured energy shift rates vs $t_{\text {laser }}$.

$6 \mathrm{eV})$. Figure 5 shows the simulated IED for nine storage times ( $t_{\text {laser }}$ from 1 to $9 \mathrm{~ms}$ ) versus the energy after subtraction of the laser photon energy $(2.214 \mathrm{eV})$. We observed a regular shift of the high-energy edge of the IED to lower energy. As we can see in Fig. 5, the maxima of the half Gaussian for each IED are slightly different. We have tried to fit all decays with the same value of the maximum but the global fit was less good than individual fits. That shows the great importance to measure precisely the relative intensities of the decays and estimate correctly the correction.

Table I gives the centers of the corresponding half Gaussians versus $t_{\text {laser }}$. These values were nicely fitted with a $t^{-\beta}$ power law $(\beta=1,1)$ showing clearly a regular shift of the IED towards low energies with time. From these values, we have estimated the energy shift rates of the high-energy part of the IED as a function of the storage time (Fig. 6). As a first approximation, the shift rate at the time $t_{\text {laser }}$ was estimated by the half of the difference between the centers of the Gaussian IED functions obtained for the previous and the next laser shots (at $t_{\text {laser }}-1 \mathrm{~ms}$ and $t_{\text {laser }}+1 \mathrm{~ms}$ ). For instance, the centers of the IEDs at $t_{\text {laser }}=1$ and $3 \mathrm{~ms}$ provided the average energy shift rate at $t=2 \mathrm{~ms}$ and so on. In Fig. 6, we remark that the energy shift rate decreases faster in the early storage time range from 2 to $4 \mathrm{~ms}$ ( 140 to $50 \mathrm{eV} / \mathrm{s}$, respectively) than at later storage time. At this point, the energy shift rate may be attributed mainly to two processes: the depletion of the high-energy part due to delayed dissociation and the cooling via the DFP. The infrared vibrational emission may contribute only marginally to the overall cooling in this time range.

In order to determine the respective contributions of delayed dissociation and cooling by DFP, the evolution of the IED was analyzed. For a given value of the internal energy (symbolized by dotted lines in Fig. 5), a population decay rate $k_{\text {pop }}$ was defined and determined by plotting the population distribution values of the IEDs as a function of $t_{\text {laser. The }}$

TABLE I. Centers of the half-Gaussian IEDs and power-law fit vs laser pulse time $t_{\text {laser }}$.

\begin{tabular}{lccccccrrr}
\hline \hline$t_{\text {laser }}(\mathrm{ms})$ & 1 & 2 & 3 & 4 & 5 & 6 & 7 & 8 & 9 \\
\hline Centers of IEDs (eV) & 6.360 & 6.229 & 6.148 & 6.079 & 5.995 & 5.974 & 5.903 & 5.893 & 5.848 \\
Power-law fit values (eV) & 6.387 & 6.219 & 6.123 & 6.055 & 6.002 & 5.960 & 5.924 & 5.893 & 5.866 \\
\hline \hline
\end{tabular}


TABLE II. Population decay rates $k_{\mathrm{pop}}\left(\mathrm{s}^{-1}\right)$, dissociation rates $k_{\text {diss }}\left(\mathrm{s}^{-1}\right)$, and $k_{\text {elec }}\left(\mathrm{s}^{-1}\right)$ electronic DFP rates vs the internal energy.

\begin{tabular}{lccc}
\hline \hline$E(\mathrm{eV})$ & $k_{\text {pop }}\left(\mathrm{s}^{-1}\right)$ & $k_{\text {diss }}\left(\mathrm{s}^{-1}\right)$ & $k_{\text {elec }}\left(\mathrm{s}^{-1}\right)$ \\
\hline 7 & 420 & 89.1 & 331 \\
6.8 & 350 & 31.4 & 319 \\
6.6 & 181 & 10.3 & 171 \\
6.4 & 110 & 3.2 & 107 \\
6.2 & 47 & 0.9 & 46 \\
6 & & 0.2 & \\
\hline \hline
\end{tabular}

extracted data were nicely fitted by an exponential decay curve, which was characteristic of the time evolution of the population of the subgroup of stored ions initially with the given particular internal energy. Repeating this procedure, we obtained the values of $k_{\text {pop }}$ listed in Table II for energy values in the range from 6.2 to $7 \mathrm{eV}$ in steps of $0.2 \mathrm{eV}$. The delayed dissociation rate $k_{\text {diss }}$ was estimated in order to determine by subtraction the electronic DFP rate $k_{\text {elec }}$. For internal energies around $7 \mathrm{eV}, k_{\mathrm{diss}}$ is not negligible and represents about $20 \%$ of the total rate, whereas at $6.2 \mathrm{eV}$ the dissociation rate can be neglected. It shows that the fluorescence cooling process is mainly responsible for the high energy shift rate of the IED as in the cases of anthracene and naphthalene cations. We interpret qualitatively the decreasing behavior of the energy shift rate as a function of time (Fig. 6) by the fact that dications of higher internal energy have a higher probability to be in one of the first electronic excited states via the IIC process because of higher level densities and consequently a higher probability to emit a visible fluorescence photon.

It is interesting to compare $k_{\text {elec }}$ rates for the cation and dication anthracene. In Ref. [12], $k_{\text {elec }}$ ranges from 430 to $25 \mathrm{~s}^{-1}$ for energy in the range from 7.4 to $6 \mathrm{eV}$ for anthracene cations. From Table II, at $7 \mathrm{eV}$ the $k_{\text {elec }}$ for anthracene dications is found to be $331 \mathrm{~s}^{-1}$, which is very close to the value of $317 \mathrm{~s}^{-1}$ for cations at the same energy. As discussed in Ref. [9], $k_{\text {elec }}$ is very sensitive to the energy and the probability of the electronic transition involved in the DFP, i.e., the $S_{2}-S_{0}$ transition for the dication and the $D_{2}-D_{0}$ transition for the cation. Higher transition energy leads to lower probability for the IIC process and therefore to a smaller value of $k_{\text {elec }}$; additionally higher probability of the electronic transition leads obviously to a larger value of $k_{\text {elec }}$. Due to the larger transition energy for the dications $(2.2 \mathrm{eV})$ than for cations $(1.7 \mathrm{eV})$, one could expect that the $k_{\text {elec }}$ would be significantly smaller for the dications. However, the transition probability is much larger for the dications than for the cations. Indeed, in Ref. [17], the transition probability of the $S_{2}-S_{0}$ transition for the dication was estimated to be twice as large as that of the $D_{2}-D_{0}$ transition for the cations. These two inverse trends provide a possible explanation for our finding that the $k_{\text {elec }}$ values are in the same order of magnitude in both cases.

\section{CONCLUSION}

We have presented laser induced neutral decays of anthracene dications stored in the Mini-Ring. The photoabsorption spectrum showed an intense line attributed to the electronic $S_{2}-S_{0}$ transition. Estimation of the time evolution of the IED has been performed in a storage time range up to $9 \mathrm{~ms}$. Electronic decay rates due to the delayed fluorescence process have been found to vary from 46 to $331 \mathrm{~s}^{-1}$ in the internal energy range from 6 to $7 \mathrm{eV}$. For comparable internal energies, the $k_{\text {elec }}$ rates of the dications of anthracene were found close to the rates of cations. This finding, which tends to show that the fluorescence cooling process would not depend strongly on the charge state, results in fact from the compensation of two opposite effects: a lower IIC probability for the dication and a higher transition rate for the $S_{2}-S_{0}$ transition (dication) than for the $D_{2}-D_{0}$ transition (cation). Further studies with higher charges or with other PAHs would be interesting in order to determine if general rules could be found concerning charge effects on the DFP. These studies may have repercussions in the studies of the composition of some regions of the interstellar medium where the presence of multiply charged PAHs may be possible.

\section{ACKNOWLEDGMENT}

This work has been supported by Agence Nationale de la Recherche (ANR) “ANNEAU” Grant No. 2010-042601.
[1] M. Goto, A. E. K. Sunden, H. Shiromaru, J. Matsumoto, H. Tanuma, T. Azuma, and K. Hansen, J. Chem. Phys. 139, 054306 (2013).

[2] A. I. S. Holm, H. Zettergren, H. A. B. Johansson, F. Seitz, S. Rosén, H. T. Schmidt, A. Ławicki, J. Rangama, P. Rousseau, M. Capron, R. Maisonny, L. Adoui, A. Méry, B. Manil, B. A. Huber, and H. Cederquist, Phys. Rev. Lett. 105, 213401 (2010).

[3] A. P. O'connor, A. Becker, K. Blaum, C. Breitenfeldt, S. George, J. Göck, M. Grieser, F. Grussie, E. A. Guerin, R. von Hahn, U. Hechtfischer, P. Herwig, J. Karthein, C. Krantz, H. Kreckel, S. Lohmann, C. Meyer, P. M. Mishra, O. Novotný, R. Repnow, S. Saurabh, D. Schwalm, K. Spruck, S. Sunil Kumar, S. Vogel, and A. Wolf, Phys. Rev. Lett. 116, 113002 (2016).
[4] Y. Toker, O. Aviv, M. Eritt, M. L. Rappaport, O. Heber, D. Schwalm, and D. Zajfman, Phys. Rev. A 76, 053201 (2007).

[5] J. U. Andersen, C. Brink, P. Hvelplund, M. O. Larsson, B. Bech Nielsen, and H. Shen, Phys. Rev. Lett. 77, 3991 (1996).

[6] V. Chandrasekaran, B. Kafle, A. Prabhakaran, O. Heber, M. Rappaport, H. Rubinstein, D. Schwalm, Y. Toker, and D. Zajfman, J. Phys. Chem. Lett. 5, 4078 (2014).

[7] L. J. Allamandola, A. G. G. M. Tielens, and J. R. Barker, Astrophys. J. 290, L25 (1985).

[8] K. Hansen, M. H. Stockett, M. Kaminska, R. F. Nascimento, E. K. Anderson, M. Gatchell, K. C. Chartkunchand, G. Eklund, H. Zettergren, H. T. Schmidt, and H. Cederquist, Phys. Rev. A 95, 022511 (2017). 
[9] P. Boissel, P. de Parseval, P. Marty, and G. Lefevre, J. Chem. Phys. 106, 4973 (1997).

[10] A. Leger, P. Boissel, F. Desert, and L. d'Hendecourt, Astron. Astrophys. 213, 351 (1989).

[11] C. Ortega, R. Bredy, L. Chen, M. Ji, J. Bernard, G. Montagne, A. R. Allouche, C. Joblin, A. Cassimi, and S. Martin, J. Phys. Conf. Ser. 583, 012038 (2015).

[12] S. Martin, M. Ji, J. Bernard, R. Brédy, B. Concina, A. R. Allouche, C. Joblin, C. Ortega, G. Montagne, A. Cassimi, Y. Ngono-Ravache, and L. Chen, Phys. Rev. A 92, 053425 (2015).

[13] J. Bernard, G. Montagne, R. Brédy, B. Terpend-Ordacière, A. Bourgey, M. Kerleroux, L. Chen, H. T. Schmidt,
H. Cederquist, and S. Martin, Rev. Sci. Instrum. 79, 075109 (2008).

[14] S. Martin, J. Bernard, R. Brédy, B. Concina, C. Joblin, M. Ji, C. Ortega, and L. Chen, Phys. Rev. Lett. 110, 063003 (2013).

[15] S. Martin, L. Chen, R. Brédy, G. Montagne, C. Ortega, T. Schlathölter, G. Reitsma, and J. Bernard, Phys. Rev. A 85, 052715 (2012).

[16] L. Chen, J. Bernard, R. Bredy, and S. Martin, J. Phys. Conf. Ser. 875, 12005 (2017).

[17] G. Malloci, C. Joblin, and G. Mulas, Chem. Phys. 332, 353 (2007). 